\title{
FRACTIONAL POWERS OF NON-NEGATIVE OPERATORS IN FRÉCHET SPACES
}

\author{
C. MARTINEZ, M. SANZ and V. CALVO \\ Universidad de Valencia \\ Facultad de Matematicas \\ Departamento de Matematica Aplicada y Astronomia \\ Burjassot, Valencia, Spain \\ (Received Apri1 21, 1988 and in revised form August 16, 1988)
}

ABSTRACT. In the present paper the theory of fractional powers, which has been restricted to date to certain operators on Banach spaces, is generalized to certain particular operators in Fréchet spaces. The main difficulty consists in the fact that. neither the holomorphic functional calculus nor the results on Banach algebras are available for bounded operators on Fréchet spaces.

All the basic properties which a good theory of fractional powers must fulfill are proved, except for the spectral relation,

$$
\sigma\left(A^{\alpha}\right)=\left\{z^{\alpha}: z \in \sigma(A)\right\}
$$

which remains an unsolved problem.

KEY WORDS AND PHRASES. Fractional powers operators. Fractional derivatives and integrals. 1980 AMS SUBJECT CLASSIFICATION CODE. 47A60, $26 \mathrm{~A} 33$.

1. INTRODUCTION AND NOTATION.

In [1] we have developed a new theory of fractional powers for non-negative operators, that is, for closed linear operators $A$ in a Banach space $X$ such that $]-\infty$, $O[$ is contained in the resolvent set $\rho(A)$ and

$$
\sup _{\lambda>0}|| \lambda(\lambda+A)^{-1}||<+\infty
$$

To this end, we have used a new definition of fractional powers which coincides with the closure of the operator $J^{\alpha}$ (defined by A.V. Balakrishnan in [2]) when $D(A)$ is dense in $X$, and is a strict extension of that operator when $A$ is not densely defined. 
The point of view introduced in [1] has proved advantageous in several aspects. First of all, it implies a substantial simplification of the theory and it shows that the denseness hypothesis on the domain $D(A)$, considered as fundamental in previous theories, is irrelevant; furthermore, it provides for the first time in the extensive literature on fractional powers, a satisfactory construction in the case of non-dense domains.

The main theorem of [1] establishes the following relation, for $\varepsilon>0$ and $\operatorname{Re} \alpha>0$ :

$$
A^{\alpha}\left[(A+\varepsilon)^{-1}\right]^{\alpha}=\left[A(A+\varepsilon)^{-1}\right]^{\alpha}
$$

In the present paper we generalize the results of [1] to operators A in Fréchet spaces that verify a condition similar to non-negativity. The essential difficulty introduced by substituting the Fréchet structure for the Banach structure consists in the loss of properties undergone by the resolvent function $z \rightarrow(z-A)^{-1}$, which is no longer analytical nor even continuous.

In 1984, Wilson Lamb [3] generalizes the concept of non-negative operators to Frechet spaces in order to obtain certain results in the field of fractional calculus. He gives a definition of fractional powers on operators, $A$, such that $(\lambda+A)^{-1}$ exists and is bounded for each $\lambda>0$, and given any seminorm, \|\|$_{p}$, among those that describe the space topology, there is another seminorm \|\|$_{q(p)}$ and a constant $C>0$ (independent of \|\|$_{p}$ ) such that for each vector $\phi$ :

$$
\left\|\left(C \lambda(\lambda+A)^{-1}\right)^{n} \phi\right\|_{p}<\|\phi\|_{q(p)} \text {, for } n=1,2, \ldots
$$

This condition assures that the resolvent function is analytical on a sector centered around the negative real semiaxis, thus allowing application of the same methods and arguments as for Banach spaces.

We propose a different kind of operator which seems more natural and strictly includes those of $W$. Lamb. Indirectly, this will be shown by means of a counterexample in which the resolvent function associated with a certain operator of our class is not analytical. The lack of this property prevents us from using or generalizing the techniques common to theories on fractional powers previousto[1]). However, we can take advantage of some of the ideas contained in [1].

Being unable to prove identity (1.1) for Frechet spaces, we have chosen to argue as follows: We show directly that

$$
D\left(A^{\alpha}\right)=D\left[(A+\varepsilon)^{\alpha}\right] \text {, for all } \varepsilon>0 \text {, }
$$

and, as a consequence, we find an integral expression for the operator

$$
A^{\alpha}-\sum_{p=0}^{n}(-1)^{p} \varepsilon^{p}\left(\begin{array}{l}
\alpha \\
p
\end{array}\right)(A+\varepsilon)^{\alpha-p}
$$


on $D(A 9)$, which will enable us to prove the essential property that a good fractional power must have, namely

$$
A^{\alpha} A^{\beta}=A^{\alpha+\beta} \quad(\text { with } \operatorname{Re} \alpha>0 \text { and } \operatorname{Re} \beta>0)
$$

On the other hand, it can be seen that the integral expression for $\left(A^{\alpha}+\mu\right)^{-1}$ and the relation

$$
(A)^{3}=A^{\alpha \beta} \quad(\text { with } 1>\alpha>0 \text { and } \operatorname{Re} B>0)
$$

established in [1] for Banach spaces, are still valid in Fréchet spaces. However, in the latter case the spectral mapping theorem

$$
\sigma\left(A^{\alpha}\right)=\left\{z^{\alpha}: z=\sigma(A)\right\}
$$

remains an open problem, at least as far as the present article is concerned.

$F$ will henceforth be a Fréchet space, and $\left\{\|\| \|_{p}{ }_{p} \geqslant 1\right.$ an increasing sequence of semi-norms defining the topology of $F$. We shall denote by $A$ a linear operator on $F$ with domain $D(A)$, and by $\rho(A)$ and $\alpha(A)$ the resolvent set and the spectrum of $A$, respectively.

The proofs of several of the results in the present paper are essentially the same as the proofs of the corresponding results in [1]. These proofs will therefore be omitred.

2. PROPERTIES OF FRACTIONAL OPERATORS.

DEFINITION 2.1. We say that $A$ is non-negative if $\rho(A) \supset\left\{\lambda e^{i \pi}: \lambda>0\right\}$ and for all $n \in N$, there exists $M_{n}>0$ and there exists $m(n) \in N$ such that

$$
\left\|\lambda(\lambda+A)^{-1} \phi\right\|_{n}<M_{n}\|\phi\|_{m(n)} \text { for all } \phi \in F \text { and for all } \lambda>0
$$

EXAMPLE 1. Given $\mathrm{p}: 1<\mathrm{p}<+\infty$, let

$F=\left\{f:\left[0,+\infty\left[+C\right.\right.\right.$ such that $\left.f\right|_{[0, a]} \in L^{P}([0, a])$, for all a $\left.>0\right\}$ with || $\mathbf{f}\left\|_{\mathrm{n}}=\left.|| \mathrm{f}\right|_{[0, \mathrm{n}]}\right\|_{\mathrm{p}}$.

Taking $A=-d / d x$ on the set

$D(A)=\left\{f \in F ;\left.f\right|_{[0, n]} \in W^{1, p}(] 0, n[)\right.$, for all $n \in N$ and $\left.f(0)=0\right\}$

it is easy to show that $A$ is a non-negative unbounded operator, which is densely defined if and only if $p<+\infty$.

EXAMPLE 2. Let $F=\left\{f \in C^{\infty}([0,1])\right.$, with $f^{(n)}(0)=0$, for $\left.n=0,1,2 \ldots\right\}$ with the seminorms

$$
\|f\|_{p}=\max _{0 \leqslant q \leqslant p}\left\|f^{(q)}\right\|_{\infty}
$$

and the (bounded) operator

$$
A=-i d / d x
$$

It can easily be shown that $A$ has an empty spectrum and that for all $\lambda>U$ and $g \in F$

$$
\left\|\lambda(\mathrm{A}+\lambda)^{-1} \mathrm{~g}\right\|_{\mathrm{p}}<2\|\mathrm{~g}\|_{\mathrm{p}+1}
$$

and thus it is a non-negative operator. 
We shall now see that for all $w: 0<w<\pi / 2$ the operator $e^{-i w_{A}}$ is not nonnegative. This will show that definition 2.1 is not equivalent to W. Lamb's definition; for if an operator satisfies (1.2), this is still true if we multiply the operator by $e^{-i w}$, if $w>0$ is small enough.

For $z=\lambda+i \sigma \lambda(\lambda>0$ and $\sigma=\operatorname{tg} w)$ and $g \in F$, we have

and consequently

$$
(A-z)^{-1} g(x)=e^{-i \lambda x+\sigma \lambda x} \int_{0}^{x} e^{i \lambda t-\sigma \lambda t} i g(t) d t
$$

$$
\left|z(A-z)^{-1} g(x)\right|=\sqrt{1+\sigma^{2}} e^{\sigma \lambda x}\left|\int_{0}^{x} e^{i \lambda t-\sigma \lambda t} g(t) d(\lambda t)\right| .
$$

Using $\quad g_{\lambda}(x)=e^{-1 \lambda x-1 / \lambda x}$, we obtain

$$
\left\|z(A-z)^{-1} g_{\lambda}\right\|_{\infty}=\sqrt{1+\sigma^{2}} e^{\sigma \lambda} \int_{0}^{\lambda} e^{-\sigma \xi-1 / \xi} \mathrm{d} \xi
$$

On the other hand,

$$
g_{\lambda}{ }^{(n)}(x)=\lambda^{n} g_{1}^{(n)}(\lambda x) \text { implies }|| g_{\lambda}{ }^{(n)} \|_{\infty}=\lambda^{n} \sup _{\tau_{\epsilon}[0, \lambda]}\left|g_{1}^{(n)}(\tau)\right|
$$

Thus

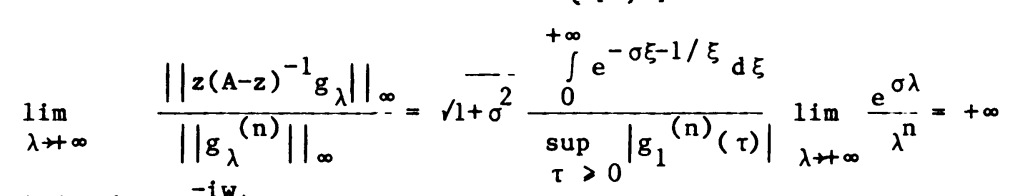

And we can conclude that $\mathrm{e}^{-i w_{A}}$ is not non-negative.

REMARK 2.1. It can also be shown that the operator A from example 2 does not satisfy (1.2) even in the case that in this condition the constant $C$ should be dependent on the seminorm \|\|$_{p}$, which is contained in the left-hand-side.

In the next definition, as well as all the other statements in this paper, we assume that $A$ is a non-negative operator.

DEFINITION 2.2. The condition that $A$ be non-negative allows us to define the integral operator $\mathrm{J}^{\alpha}$ in the same way as it was defined in [1] for Banach spaces, and the following property holds:

$$
\mathrm{J} \alpha^{\beta}=\mathrm{J}^{\alpha+\beta} \quad \text { (with } \operatorname{Re} \alpha>0 \text { and } \operatorname{Re} \beta>0 \text { ). }
$$

If $A$ is continuous, we define

$$
A^{\alpha}=J^{\alpha}
$$

If $A$ is not continuous and $0 \in \rho(A)$, we have that the operator $A^{-1}$ is a continuous non-negative operator, since

(with $k(n)=\max \{n, m(n)\}$ ) for all $\lambda>0$ and $\phi \in F$.

$$
\begin{aligned}
\left\|\lambda\left(A^{-1}+\lambda\right)^{-1} \phi\right\|_{n} & =\left\|A\left(A+\lambda^{-1}\right)^{-1} \phi||_{n}=\right\| \phi-\lambda^{-1}\left(A+\lambda^{-1}\right)^{-1} \phi \|_{n}< \\
& <\|\phi\|_{n}+M_{n}\|\phi\|_{m(n)}<\left(1+M_{n}\right)\|\phi\|_{k(n)}
\end{aligned}
$$


$\left(A^{-1}\right)^{\alpha}$ is a one-to-one operator, since if $n>\operatorname{Re} \alpha$, we have $\left(A^{-1}\right)^{n-\alpha}\left(A^{-1}\right)^{\alpha}=\left(A^{-1}\right)^{n}$,

and consequent.ly:

$$
\left(A^{-1}\right)^{\alpha} \phi=0 \Rightarrow\left(A^{-1}\right)^{n} \phi=0 \Rightarrow \phi=0 .
$$

We define

$$
A^{\alpha}=\left(\left(A^{-1}\right)^{\alpha}\right)^{-1}
$$

It is easy to prove that, for $0<\operatorname{Re} \alpha<1$ and $\phi \in D(A)$

$$
\left(\left(A^{-1}\right)^{\alpha}\right)^{-1} \phi=\frac{\sin \alpha \pi}{\pi} \int_{0}^{+\infty} \lambda^{\alpha-1}(\lambda+A)^{-1} A \phi d \lambda .
$$

Finally, if $A$ is not continuous and $0 \notin \rho(A)$, we define

$$
A^{\alpha} \phi=\lim _{\varepsilon \rightarrow 0}(A+\varepsilon)^{\alpha}{ }_{\phi}
$$

on the vectors $\phi$ for which the limit exists.

REMARKS. i) From (2.2) and (2.3) it follows, by direct composition of the powers (or their inverses) in their integral form, as shown in [2], that

$$
A^{\alpha} A^{\beta}=A^{\alpha+\beta} \quad(\text { with } \operatorname{Re} \alpha>0 \text { and } \operatorname{Re} \beta>0 \text { ) }
$$

when $A$ is continuous, and $A$ not being continuous, when $0 \notin \rho(A)$.

ii) Arguing as in [1] it can be proved that when $A$ is not continuous, $J^{\alpha}$ is a closable operator and $A^{\alpha}$ is an extension of the closure of $J^{\alpha}$.

The subsequent results will give a more precise meaning to the definition of $A^{\alpha}$ in the more general case in which $0 \notin \rho(A)$ and $A$ is not continuous.

PROPOSITION 2.1. When $A$ is not continuous, the domain of $(A+\varepsilon)^{\alpha}$ is independent of $\varepsilon>0$ (and equal to $D(A)$ if $0 \in \rho(A)$ ).

PROOF. We first assume $0<\operatorname{Re} \alpha<1$. Starting with the identity

$$
D[(A+\varepsilon) \alpha]=R\left(\left[(A+\varepsilon)^{-1}\right] q\right)
$$

and using the integral expression of the operator $\mathrm{J}^{\alpha}$ associated with the operator $(A+\varepsilon)^{-1}$, we obtain

$$
D[(A+\varepsilon) q]=\left\{\int_{0}^{+\infty} \lambda^{-\alpha}(\lambda+\varepsilon+A)^{-1} \phi d \lambda: \phi \in F\right\} \text {, for all } \varepsilon>0 \text {. }
$$

On the other hand, given $\varepsilon, \eta>0$ the resolvent formula leads to

$$
\begin{aligned}
& \int_{0}^{+\infty} \lambda^{-\alpha}(\lambda+\varepsilon+A)^{-1} \phi d \lambda-\int_{0}^{+\infty} \lambda^{-\alpha}(\lambda+n+A)^{-1} \phi d \lambda= \\
& =(n-\varepsilon) \int_{0}^{+\infty} \lambda^{-\alpha}(\lambda+\varepsilon+A)^{-1}(\lambda+n+A)^{-1} \phi d \lambda, \text { for all } \phi \in F,
\end{aligned}
$$


and the operator $A$ can be introduced into the integrand on the right-hand side with no influence on the convergence of the integral; for this reason

$$
\left[(A+\varepsilon)^{-1}\right]_{\phi}-\left[(A+\eta)^{-1}\right] \alpha_{\phi \in D(A)},
$$

and thus

$$
\left[(A+\varepsilon)^{-1}\right]_{\phi}^{\alpha_{\phi}}\left[(A+\eta)^{-1}\right]_{\phi \in D}^{\alpha_{\phi}}\left[(A+\varepsilon)^{\alpha} \cap \cap\left[(A+n)^{\alpha}\right]\right.
$$

for all $\phi \in F$, so we get

$$
D\left[(A+\varepsilon)^{\alpha}\right]=D[(A+n) \text {. }
$$

Next we shall prove by induction on $n$ that, for $0<\operatorname{Re} \alpha<2^{n}$, there exists $B(\alpha)$ with $\operatorname{Re} B(\alpha)>\operatorname{Re} \alpha$, such that for all $\phi \in F$

$$
\left[(A+\varepsilon)^{-1}\right]_{\phi}-\left[(A+n)^{-1}\right] \alpha_{\phi \in D}\left[(A+\varepsilon)^{\beta(\alpha)}\right] \cap D\left[(A+n)^{\beta(\alpha)}\right]
$$

which will imply the equality of the domains $D\left[(A+\varepsilon)^{\alpha}\right]$ and $D\left[(A+\eta)^{\alpha}\right]$.

This result has already been proved for $n=0$. Assuming that it holds up to $n-1$, take $\alpha$ such that $0<\operatorname{Re} \alpha<2^{n}$. We have

$$
\left[(A+\varepsilon)^{-1}\right]_{\phi}-\left[(A+\eta)^{-1}\right]_{\phi}^{\alpha_{\phi}}=\left[(A+\varepsilon)^{-1}\right]^{\alpha / 2} \psi+\left[(A+\eta)^{-1}\right]^{\alpha / 2} \psi
$$

with

$$
\psi=\left[(A+\varepsilon)^{-1}\right]^{\alpha / 2} \phi-\left[(A+n)^{-1}\right]^{\alpha / 2} \phi
$$

and this element belongs to $D\left[(A+\varepsilon)^{\beta(\alpha / 2)}\right] \cap D\left[(A+\eta)^{\beta(\alpha / 2)}\right]$ by the induction hypothesis.

From (2.6) it is easily concluded that $\left[(A+\varepsilon)^{-1}\right]^{\alpha / 2} \psi$ and $\left[(A+n)^{-1}\right]^{\alpha / 2} \psi$ belong to the set

$$
D\left[(A+\varepsilon)^{\alpha / 2}+B(\alpha / 2)\right] \cap D\left[(A+\eta)^{\alpha / 2+B(\alpha / 2)}\right]
$$

and relation (2.5) is established (with $B(\alpha)=\alpha / 2+\beta(\alpha / 2)$ ).

If $0 \in \rho(A)$, then $A^{\alpha}$ is defined in the same way as $(A+\varepsilon)^{\alpha}$, and therefore the argument is still valid if the condition $\eta>0$ is substituted by $n=0$. This yields $D[(A+\varepsilon) q=D(A)$.

PROPOSITION 2.2. When $A$ is not continuous we denote by $D_{\alpha}$ the set $D[(A+\varepsilon) q$. Then the function

$$
h(t)=(A+t)^{\alpha_{\phi}},
$$

with $₫ \in D_{\alpha}$ and $t>0$, is Holder continuous in each bounded interval $\left.] 0, a\right]$, with exponent $\operatorname{Re} \alpha / 2^{n}$, as long as $0<\operatorname{Re} \alpha<2^{n}$. Consequently

$$
\lim _{\varepsilon \rightarrow 0}(A+\varepsilon)^{\alpha_{\phi}}=A^{\alpha}{ }_{\phi}
$$

and $D(A)=D_{\alpha}$. 
PROOF. We have for $0<\operatorname{Re} \alpha<1$ and $\phi D(A)$

$$
\begin{aligned}
& (A+\varepsilon)^{\alpha_{\phi}}-(A+\eta)^{\alpha_{\phi}}=\frac{\sin \alpha \pi}{\pi}\left[\int_{0}^{\delta} \lambda^{\alpha-1}\left(-\lambda(A+\varepsilon+\lambda)^{-1} \phi+\lambda(A+n+\lambda)^{-1} \phi\right) d \lambda+\right. \\
& \left.+\int_{\delta}^{+\infty} \lambda^{\alpha}(\varepsilon-\eta)(A+\varepsilon+\lambda)^{-1}(A+n+\lambda)^{-1} \phi d \lambda\right]
\end{aligned}
$$

using the identity $(A+\varepsilon)(A+\varepsilon+\lambda)^{-1} \phi=\phi-\lambda(A+\varepsilon+\lambda)^{-1} \phi$ and the resolvent formula. The two integrals are absolutely convergent, for all $\phi \in F$.

Applying the formula (2.7) to the element $(A+1)^{-1} \phi$, for all $\phi \in D_{\alpha}$ and taking into account that the one-to-one operator $(A+1)^{-1}$ can be placed on the left, since it commutes with the integral sign we conclude that (2.7) holds for all $\phi \in D_{\alpha}$. Taking $\delta=|\varepsilon-\eta|$, we have

$$
\begin{aligned}
& \|(A+\varepsilon)^{\alpha} \phi-(A+n)^{\alpha} \phi||<\left|\frac{s i n \alpha \pi}{\pi}-\right|\left[\int_{0}^{n-\varepsilon \mid} \lambda^{\operatorname{Re} \alpha-1} 2 M_{n}\|\phi\|_{m(n)} d \lambda+\right. \\
& \left.\quad+\int_{n-\varepsilon \mid}^{+\infty} \lambda^{\operatorname{Re} \alpha-2}|n-\varepsilon| M_{n} M_{m(n)}\|\phi\|_{m(m(n))} d \lambda\right]
\end{aligned}
$$

Hence

$$
\|(A+\varepsilon)^{\alpha}{ }_{\phi-(A+n)} \alpha_{\phi}||<\left|\frac{\sin \alpha \pi}{\pi}\right||n-\varepsilon|^{\operatorname{Re} \alpha}\left[\frac{2 M_{n}}{\operatorname{Re} \alpha}\|\phi\|_{m(n)}+\frac{M_{n} M_{m(n)}}{\operatorname{Re} \alpha-1} \|\left.\phi\right|_{m(m(n))}\right]
$$

and consequently $h(t)=(A+t) \alpha_{\phi}$, with $\phi \in D_{\alpha}$ and $t>0$ is Hölder continuous with exponent $\operatorname{Re} \alpha$, so $h$ is continuous and

$\lim _{\varepsilon \neq 0}(A+\varepsilon)^{\alpha}$

Arguing by induction, it is easy to show that in each bounded interval $] 0, a]$ is a Holder continuous with exponent $\operatorname{Re} \alpha 2^{n}$ with $n \in N$ such that $0<\operatorname{Re} \alpha<2^{n}$. For $n=0$ this statement has actually been proved, and assuming it holds up to n-1 and $\alpha$ such that $0<\operatorname{Re} \alpha<2^{n}$, then the relation

with

$$
||(A+\varepsilon)^{\alpha}-(A+\eta)^{\alpha} \|_{1}|=|\left|(A+\varepsilon)^{\alpha / 2} \psi-(A+\eta)^{\alpha / 2} \psi\right| \mid
$$

$$
\psi=(A+\varepsilon)^{\alpha / 2} \phi+(A+\eta)^{\alpha / 2} \phi
$$

implies, by the induction hypothesis, the property that was claimed for h.

Therefore $h$ is continuous and converges as $t$ tends to zero. Thus, if $A$ is not continuous and $0 \notin \rho(A)$, by the definition of $D(A)$, this set is equal to $D_{\alpha}$. On the other hand, if $0 \in \rho(A)$, both domains being equal by Proposition 2.1., we also conclude that the following equation holds:

$$
\lim _{\varepsilon \rightarrow 0}(A+\varepsilon)^{\alpha} \phi=A_{\phi, \text { for all } \phi \in D(A)^{\alpha}}^{\alpha} \text {. }
$$


REMARK. Relation (2.8) is obtained as in the classical theory if $A$ is continuous.

LEMMA 2.1. Given $p \in N$, there exists $q \in N$ and there exists $K>0$ such that $\left\|\left[(A+\varepsilon)^{-1}\right] \alpha_{\phi}\right\|_{p}<K \varepsilon^{-\operatorname{Re} \alpha}\|\phi \mid\|_{q}$, for all $\varepsilon>0$ and for all $\psi<F$.

PROOF. The case $0<\operatorname{Re} \alpha<1$ is immediate from the integral expression for $\left[(A+\varepsilon)^{-1}\right]^{\alpha}$, which yields

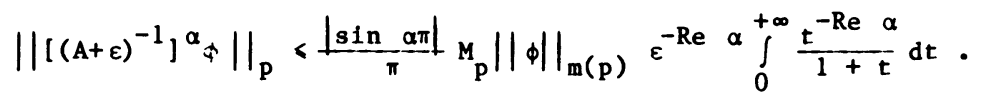

The general case is reduced to the former by repeatedly applying (2.9) after writing

$$
\left[(A+\varepsilon)^{-1}\right]^{\alpha}=\left(\left[(A+\varepsilon)^{-1}\right]^{\alpha / n}\right)^{n}
$$

with a natural number $n$ such that $0<\operatorname{Re} \alpha / n<1$.

PROPOSITION 2.3. Given $\phi \in D(A), n \in N$ and $\varepsilon>0$, the integral

$$
\int_{0}^{1} t^{n}(A+\varepsilon t)^{\alpha-(n+1)} \phi d t
$$

(which may be improper at $t=0$ ) converges and, furthermore,

$$
\begin{aligned}
& A^{\alpha}{ }_{\phi}-\sum_{p=0}^{n}(-1)^{p} \varepsilon^{p}\left(_{p}^{\alpha}\right)(A+\varepsilon)^{\alpha-p}{ }_{\phi}= \\
& =(-1)^{n+1} \varepsilon^{n+1}\left({ }_{n}^{\alpha}\right)(\alpha-n) \int_{0}^{1} t^{n}(A+\varepsilon t)^{\alpha-(n+1)} \phi d t
\end{aligned}
$$

where by $(A+\varepsilon)^{\alpha-p}$ we mean $\left[(A+\varepsilon)^{-1}\right]^{p}(A+\varepsilon)^{\alpha}$.

PROOF. We argue as in the corresponding Proposition of [1]: first we show that the function $h$ of Proposition 2.2, is indefinitely differentiable, then we write the Taylor expansion of $h(h)$ with respect to $h(\varepsilon)$. In the resulting expression we find that we can take the limit for $n$ tending to zero, which leads to (2.10). Only slight changes must be made in order to make up for the differences between Banach and Fréchet spaces, so we omit the details.

3. MAIN RESULTS.

LEMMA 3.1. Given $\alpha, \beta \in C, n \in M$ and $\varepsilon>0$, with $n+1>\operatorname{Re} \alpha, \operatorname{Re} \beta>0$ and $\phi \in D[(A+\varepsilon) \propto$, we have

and

$$
\int_{0}^{1} t^{n}(A+\varepsilon t)^{\alpha-(n+1)} \phi d t \in D\left[(A+\varepsilon)^{\beta}\right]
$$

$$
\lim _{\varepsilon \rightarrow 0} \varepsilon^{n+1}(A+\varepsilon)^{\beta} \int_{0}^{1} t^{n}(A+\varepsilon t)^{\alpha-(n+1)} \phi d t=0
$$

PROOF. As the operator $(A+\varepsilon)^{\beta}$ is closed, in order to prove that the integral belongs to its domain, it suffices to show that this fact is true for the integrand, and that the following integral (which is improper at $t=0$ if $0 \notin \rho(A)$ ) 
is convergent. $\int_{0}^{1} t^{n}(A+\varepsilon)^{\beta}(A+\varepsilon t)^{\alpha-(n+1)} d d t$

The first assertion is obvious, since $n+1>\operatorname{Re} B$.

We will show that (3.1) converges with respect to any seminorm \|\|$_{p}$. To this end, we write

$$
(A+\varepsilon)^{\beta}(A+\varepsilon t)^{\alpha-(n+1)} \phi=\left[(A+\varepsilon)^{-1}\right]^{n+1-\beta_{\psi}}
$$

with

$$
\psi=(A+\varepsilon)^{n+1}(A+\varepsilon t)^{\alpha-(n+1)} \phi .
$$

Then, by Lemma 2.1 , there exists $q \in N$ and $K>0$ such that

$$
\left\|\left[(A+\varepsilon)^{-1}\right]^{n+1-\beta} \psi\right\|_{p}<K\|\psi\|_{q} \varepsilon^{\operatorname{Re} \beta-(n+1)}
$$

In order to estimate $\|\psi\|_{q}$ by means of a suitable function of $t$ we use the following decomposition:

$$
\begin{aligned}
& (A+\varepsilon)^{n+1}\left[(A+\varepsilon t)^{-1}\right]^{n+1}(A+\varepsilon t)^{\alpha_{\phi}}= \\
& =\sum_{i=0}^{n+1}\left(\begin{array}{c}
n+1 \\
i
\end{array}\right) \varepsilon^{i}(1-t)^{i}\left[(A+\varepsilon t)^{-1}\right]^{i}(A+\varepsilon t)^{\alpha}{ }_{\phi} .
\end{aligned}
$$

Applying Lemma 2.1 to the last term of the sum and considering the continuity of $(A+\varepsilon t)^{\alpha}$ as $t$ ranges over the interval $[0,1]$, we conclude that there exists $H_{1}$ and $H_{2}$, positive and independent of $t$, such that

$$
\|\psi\|_{q}<H_{1} \sum_{i=0}^{n}(1-t)^{i} t^{-i}+H_{2} \varepsilon^{R e} \alpha_{(1-t)^{n+1}} t^{R e} \alpha-(n+1) .
$$

This implies convergence of the integral (3.1). Besides, the estimation of the integrand also implies that

as claimed.

$$
\lim _{\varepsilon \rightarrow 0} \varepsilon^{n+1}(A+\varepsilon)^{\beta} \int_{0}^{1} t^{n}(A+\varepsilon t)^{\alpha-(n+1)} \phi d t=0
$$

LEMMA 3.2. If $P$ is a polynomial with complex coefficients and such that all its roots are non-zero, then if $\phi \in F$ satisfies

we have $\phi \in D\left[(A+\varepsilon)^{\alpha}\right]$.

$$
P\left[(A+\varepsilon)^{-1}\right] \quad \phi \in D\left[(A+\varepsilon)^{\alpha}\right]
$$

PROOF. Let $r_{1}, r_{2}, \ldots r_{h}$ be the roots of $P$, and $k_{1}, k_{2}, \ldots k_{h}$ their respective multiplicities. Then the hypothesis can be rewritten as

$$
\left[(A+\varepsilon)^{-1}-r_{1}\right] \psi \in D\left[(A+\varepsilon)^{\alpha}\right]
$$

with

$$
\psi=\left[(A+\varepsilon)^{-1}-r_{1}\right]^{k_{1}-1} \pi_{2 \varangle i \varangle}\left[(A+\varepsilon)^{-1}-r_{i}\right]^{k_{i}}
$$


and, if we show that $\psi \in D\left[(A+\varepsilon)^{\alpha}\right]$, the argument can be repeated until we arrive at the element $\phi$.

Taking a natural number m such that $2^{m}>\operatorname{Re} \alpha,(3.2)$ implies that.

$$
\left.\left(_{p=0}^{m-1}\left[(A+\varepsilon)^{-1}\right]^{2^{p}}+r^{2^{p}}\right)\right]\left[(A+\varepsilon)^{-1}-r_{1}\right] \psi \in D\left[(A+\varepsilon)^{\alpha}\right] .
$$

Now, carrying out the operation on the left-hand side, we get

$$
\left[(A+\varepsilon)^{-1}\right]^{2^{m}} \psi-r_{1}^{2^{m}} \psi \in D\left[(A+\varepsilon) a_{0}\right.
$$

Since $2^{m}>\operatorname{Re} \alpha$, we have

$$
\left[(A+\varepsilon)^{-1}\right]^{2^{m}} \psi \in D[(A+\varepsilon) q,
$$

and we obtain $\psi \in D\left[(A+\varepsilon)^{\alpha}\right]$ as claimed.

PROPOSITION 3.1. If $\operatorname{Re} \alpha>0$ and $\operatorname{Re} \beta>0$, the following equality holds.

$$
A^{\beta} A^{\alpha}=A^{\alpha+\beta} \text {. }
$$

PROOF. We shall see that the operator $A^{\alpha+\beta}$ is an extension of $A^{\beta_{A}}{ }^{\alpha}$. Let $\phi \in D\left(A^{\alpha}\right)$ such that $A^{\alpha} \phi \in D\left(A^{\beta}\right)$. According to $(2.10)$ we have for $a 11 n \in N$ and for all $\varepsilon>0$ :

$$
\begin{aligned}
& A^{\alpha} \alpha_{p=0}^{n}(-1)^{p} \varepsilon^{p}\left(\sum_{p}^{\alpha}(A+\varepsilon)^{\alpha-p} \phi+\right. \\
& +(-1)^{n+1} \varepsilon^{n+1}\left({ }_{n}^{\alpha}\right)(\alpha-n) \int_{0}^{1} t^{n}(A+\varepsilon t)^{\alpha-(n+1)} \phi d t .
\end{aligned}
$$

From here, taking $n+1>\operatorname{Re} \alpha, \operatorname{Re} \beta$ and applying Lemmas 3.1 and 3.2 it follows that $(A+\varepsilon)^{\alpha} \phi \in D\left[(A+\varepsilon)^{\beta}\right]$, that is $\phi \in D\left(A^{\alpha+\beta}\right)$.

In order to prove that $A^{\beta} A^{\alpha} \phi=A^{\alpha+\beta_{\phi}}$ we let $(A+\varepsilon)^{\beta}$ operate on both sides of (3.3) and take limits for $\varepsilon$ tending to zero. By Lemma 3.1 we know that

$$
\lim _{\varepsilon \rightarrow 0} \varepsilon^{n+1}(A+\varepsilon)^{\beta} \int_{0}^{1} t^{n}(A+\varepsilon t)^{\alpha-(n+1)} \phi d t=0
$$

so, in order to establish the equality of $A^{\beta} A^{\alpha} \phi$ and $A^{\alpha+\beta} \phi$, it will suffice to prove that

$$
\lim _{\varepsilon \rightarrow 0}(A+\varepsilon)^{\beta}\left(\varepsilon^{i}(A+\varepsilon)^{\alpha-1} \phi\right)=0 \quad(1<i<n)
$$

To this end, let $\delta$ be such that $0<\delta<\min \{1, \operatorname{Re}(\alpha+\beta)\}$. Using Lemma 2.1 , we have for all $p \in N$,

$$
\begin{aligned}
& \left.\left\|\left.(A+\varepsilon)^{\beta}(A+\varepsilon)^{\alpha-i} \phi\right|_{p}=\right\|[A+\varepsilon)^{-1}\right]^{i-\delta}(A+\varepsilon)^{\alpha+\beta-\delta} \phi||_{p} \\
& <K \varepsilon^{\delta-i}||(A+\varepsilon)^{\alpha+\beta-\delta_{\phi}} \|_{q},
\end{aligned}
$$


where $K$ and $q$ do not depend on $\varepsilon$ and the term within the seminorm is continuous in a neighborhood of zero owing to the existence of the limit

$$
\lim _{\varepsilon \neq 0}(A+\varepsilon)^{\alpha+\beta-\delta_{\phi}=A^{\alpha+\beta-\delta_{\phi}}}
$$

(This limit exists because $\phi \in D\left[(A+\varepsilon)^{\alpha+\beta}\right]$, a set which is included in $\left.D\left[(A+\varepsilon)^{\alpha+\beta-\delta}\right]=D\left(A^{\alpha+\beta-\delta}\right)\right)$. Thus we have proved $(3.4)$, as intended.

Next we shall see that $A^{\beta} A^{\alpha}$ is an extension of $A^{\alpha+\beta}$.

Let $\phi \in D\left(A^{\alpha+\beta}\right)$. Then $\phi \in D\left[(A+\varepsilon)^{\alpha+\beta}\right]$ and consequently $\phi \in D(A q)$ and $A^{\alpha} \phi$ can be expressed as in (3.3). Now we argue as in the proof of the inclusion $A^{\alpha}+\beta_{\supset A} \beta_{A} \alpha$, except that 1 it is now the second member of (3.3), term by term, of which we know beforehand that it belongs to $D\left[(A+\varepsilon)^{\beta}\right]$. Thus we have $A^{\alpha} \phi E D\left[(A+\varepsilon)^{\beta}\right]$ and taking limits we obtain

$$
A^{\beta_{A}}{ }^{\alpha}=A^{\alpha+\beta_{\phi}} \text {. }
$$

PROPOSITION 3.2. $A^{\alpha}$ is a closed operator.

PROOF. Let $\left\{\phi_{p}\right\}_{p} \geqslant 1$ be a sequence in $D(A)$ such that the following limits exist:

$$
\begin{aligned}
& \lim _{p \rightarrow \infty} \phi_{p}=\phi \\
& \lim _{p \rightarrow \infty} A^{\alpha} \phi_{p}=\psi
\end{aligned}
$$

Let $n \in N$ such that $n>\operatorname{Re} \alpha$ and $\mu>0$. Since $A^{\alpha}$ is an extension of the closable operator $J^{\alpha}$ and $D(J) \supset D\left(A^{n}\right)$, we have

$$
A^{\alpha}\left[(A+\mu)^{-1}\right]_{\phi}^{n_{1}}=\left[(A+\mu)^{-1}\right]^{n_{\psi}}
$$

Consequently, as by additivity (Proposition 3.1) we know that $D\left(A^{n-q} \supset D\left(A^{n}\right)\right.$, we arrive at

and

$$
A^{\alpha}\left[(A+\mu)^{-1}\right]^{n} \quad \phi \in D\left(A^{n-\alpha}\right)
$$

$$
A^{n}\left[(A+\mu)^{-1}\right]^{n} \quad=A^{n-\alpha}\left[(A+\mu)^{-1}\right]^{n} \psi
$$

from which it follows, repeating the argument,

$$
A^{n}\left[(A+\mu)^{-1}\right]^{n} \quad \phi \in D\left(A^{\alpha}\right)
$$

Now this implies, by Lemma 3.2 that $\psi \in D\left(A^{\alpha}\right)$ and taking this into (3.6), it follows that

$$
\mathrm{A}^{\alpha} \phi=\psi
$$

thus $A^{\alpha}$ is closed. 
The following Proposition can be proved by means of the techniques developed in [1] and [2].

PROPOSITION 3.3. We have. (i) When $A$ is not continuous, $A^{\alpha}$ is an extension of the closure operator $\mathrm{J}^{\alpha}$, and the equality holds if and only if $A$ is densely defined.

(ii) If $\mu$ belongs to the non-empty set

$$
\left\{\mu \text { C } \sim\{0\}: \mu+\lambda^{\alpha} e^{i \theta \alpha} \neq 0 \text { with } \lambda>0, y-\pi \leq \theta \leq \pi\right\}
$$

then $A^{\alpha}+\mu$ has a continuous inverse given by the convergent integral

$$
2 \frac{1}{2 \pi} \int_{0}^{+}\left[-\frac{1}{\mu+\lambda^{\alpha} e^{-i \pi \alpha}}-\frac{1}{\mu+\lambda^{\alpha} e^{i \pi \alpha}}\right](A+\lambda)^{-1} d \lambda .
$$

(iii) If $0<\alpha<1$, then $A^{\alpha}$ is non-negative and we have

$$
\left(A^{\alpha}\right)^{\beta}=A^{\alpha \beta} \text {, for all } \beta \text { with } \operatorname{Re} \beta>0 \text {. }
$$

REMARK 3.1. The concept of a non-negative operator, as well as the results in this paper, can easily be generalized to complete locally convex spaces.

\section{REFERENCES}

1. MARTINEZ, C., SANZ, M., MARCO, M., Fractional powers of operators, J. Math. Soc. of Japan (1988), $40(2)$.

2. BALAKRISHNAN, A.V., Fractional powers of closed operators and the semigroups generated by them, Pacific J. Math. 10 (1960), 419-437 (MR 22, 5899).

3. LAMB, W., Fractional powers of operators defined on a Fréchet space, Proc. Edinburgh Math. Soc. 27, (1984), 165-180.

4. FATTORINI, H.0., The Cauchy problem. Encyclopedia of Mathematics and its. applications. Vo1. 18, Addison-Wesley, Reading, Mass. (1983).

5. FATTORINI, H.0., Second-Order linear Differential Equations in Banach spaces, North-Holland (1985).

6. HOVEL, H.W. and WESTPHAL, U., Fractional powers of closed operators, Studia Math. 42, (1972), 177-194 (MR 46, 2481).

7. KOMATSU, H., Fractional powers of operators, Pacific J. Math. 19, (1966), 285-346 (MR 34, 1862).

8. KRASNOSEL'SKII, M.A. and SOBOLEVSKII, P.E., Fractional powers of operators acting in Banach spaces, Doclady Acad. Nank SSS 129, (1959), 499-502.

9. MCBRIDE, A.C. and ROACH, G.F., Fractional calculus, Research notes in Mathematics 138, Pitman, Advanced Publishing Program 38 (1985).

10. WATANABE, J., On some properties of fractional powers of linear operators, Proc. Japan Acad. 37, (1961), 273-275. 


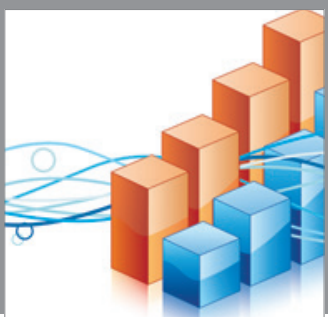

Advances in

Operations Research

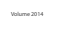

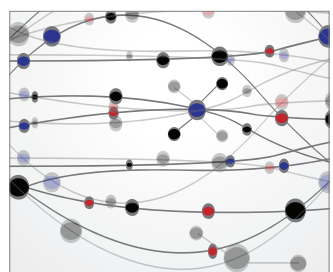

\section{The Scientific} World Journal
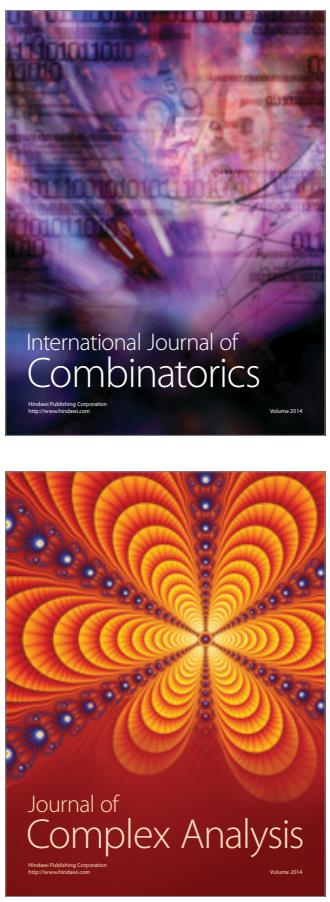

International Journal of

Mathematics and

Mathematical

Sciences
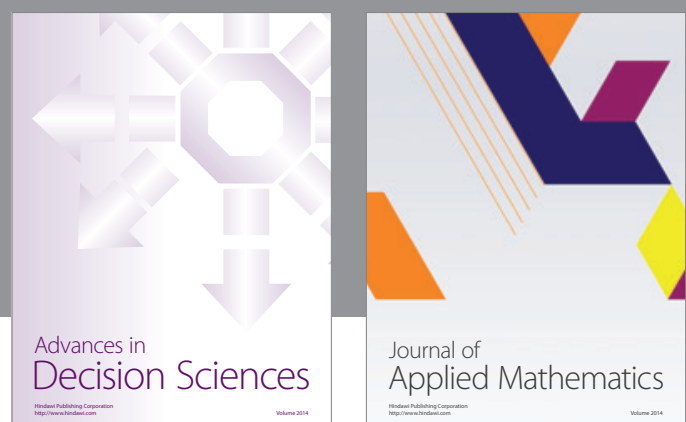

Journal of

Applied Mathematics
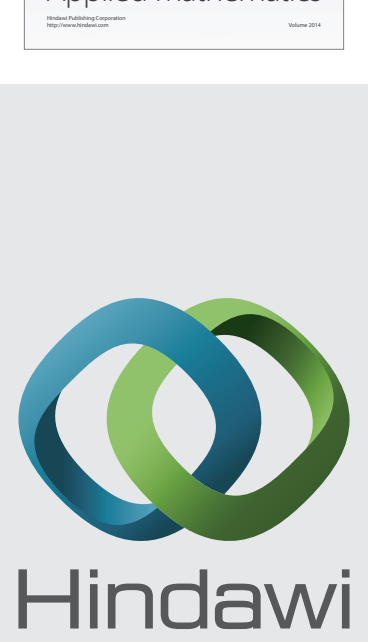

Submit your manuscripts at http://www.hindawi.com
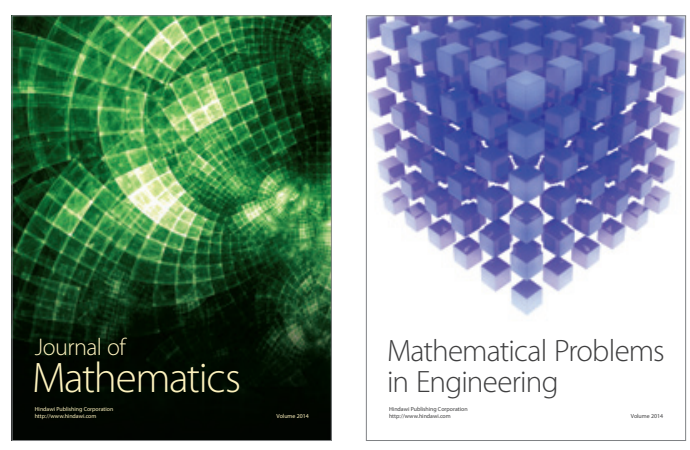

Mathematical Problems in Engineering
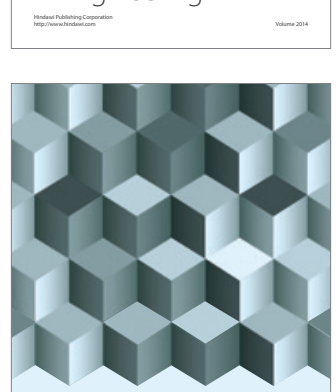

Journal of

Function Spaces
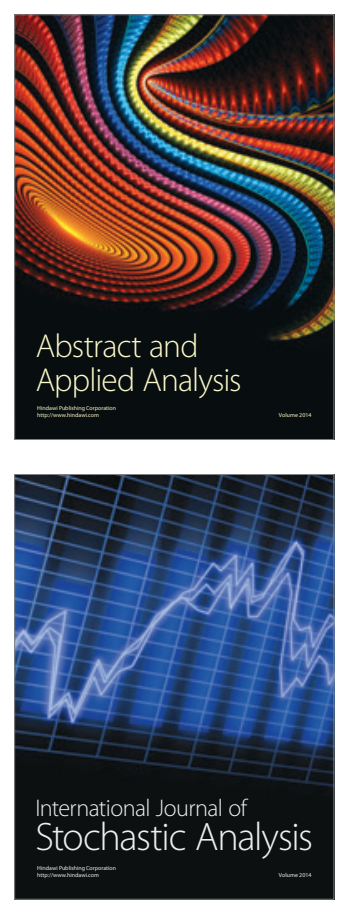

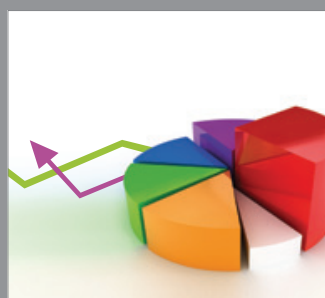

ournal of

Probability and Statistics

Promensencen
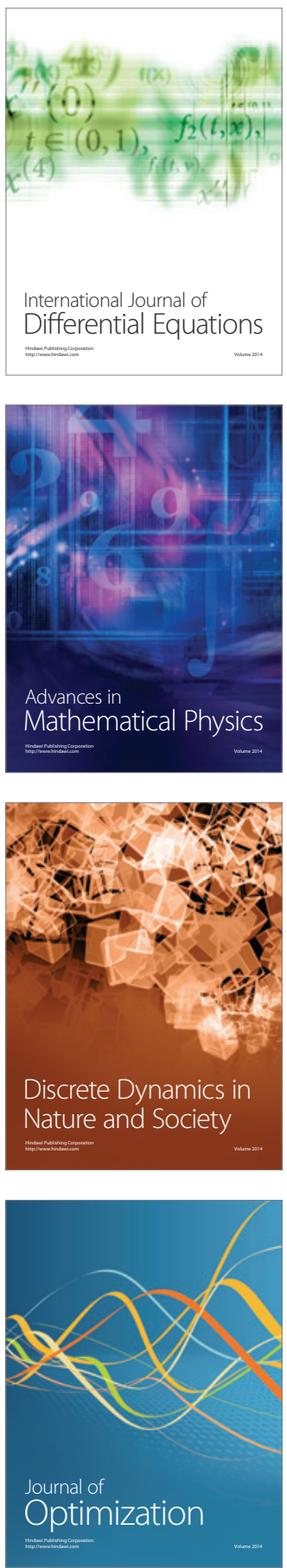